\title{
How to Perform and Interpret Timed Barium Esophagogram
}

\author{
Zafar Neyaz, ${ }^{1 *}$ Mahesh Gupta $^{2}$ and Uday C Ghoshal ${ }^{2}$ \\ Departments of ${ }^{1}$ Radiodiagnosis and ${ }^{2}$ Gastroenterology, Sanjay Gandhi Postgraduate Institute of Medical Sciences, Lucknow, India
}

Timed barium esophagogram (TBE) is a simple and objective method for assessing the esophageal emptying. The technique of TBE is similar to usual barium swallow with some modifications, which include taking multiple sequential films at pre-decided time interval after a single swallow of a fixed volume of a specific density barium solution. While many authors have used height and width of the barium column to assess the esophageal emptying, others have used the area of the barium column. TBE is being used in patients with suspected or confirmed achalasia and to follow-up those who have been treated with pneumatic dilation or myotomy. This review discusses technique of performing TBE, interpretation and its utility in clinical practice.

(J Neurogastroenterol Motil 2013;19:251-256)

Key Words

Esophageal achalasia; Esophageal motility disorders; Esophageal sphincter, lower; Esophagus

\section{Introduction}

Radiological evaluation of the esophagus has the ability to diagnose both structural changes as well as motility disorders. ${ }^{1}$ While barium esophagography is performed for the detailed evaluation of the esophagus, a timed barium esophagogram (TBE) is performed specifically to study the esophageal emptying. ${ }^{2}$ TBE quantifies the esophageal emptying easily and accurately, and is essential in the initial evaluation of motor dysphagia, commonly caused by achalasia. ${ }^{3-5}$ Esophageal scintigraphy, endoscopic evaluation, conventional and high resolution manometry are the other techniques for evaluating esophageal anatomy and functions. ${ }^{6-8}$ However, scintigraphy and manometry are not widely available and endoscopy is not a good modality for the functional assessment of esophagus. Scintigraphy technique varies considerably in different institutions including consistency of the radionuclide meal. ${ }^{3}$ Barium studies are cheaper and readily available. Various authors have used TBE in predicting response to pneumatic dilation (PD) but the technique and interpretation has been variable. Moreover, criteria to define response after PD according to esophageal emptying as seen in TBE also need standardization. This review discusses technique of performing TBE, interpretation and its utility in clinical practice.

Received: February 24, 2013 Revised: March 21, 2013 Accepted: March 23, 2013

(c) This is an Open Access article distributed under the terms of the Creative Commons Attribution Non-Commercial License (http://creativecommons. org/licenses/by-nc/3.0) which permits unrestricted non-commercial use, distribution, and reproduction in any medium, provided the original work is properly cited.

*Correspondence: Zafar Neyaz, MD

Assistant Professor, Department of Radiodiagnosis, Sanjay Gandhi Postgraduate Institute of Medical Sciences, Lucknow 226014, India

Tel: +91-800-490-4488, Fax: +91-522-266-8017 (or 8078),E-mail: zafar@sgpgi.ac.in

Financial support: None.

Conflicts of interest: None. 


\section{Technique}

The technique of TBE is similar to the usual barium swallow with some modifications, which include taking multiple sequential films at pre-decided time interval after a single swallow of a fixed volume of a barium suspension of specific density. Several techniques of TBE have been described in the literature by various authors with minor variations. ${ }^{3-5}$ Patients are advised overnight fasting prior to TBE. ${ }^{9}$ Entire study is performed in the erect posture using standard radiography machine. A low density barium sulphate suspension ( $45 \%$ weight by volume) is ingested orally within 15-20 seconds (Fig. 1). The volume of suspension (usually 100 to $250 \mathrm{~mL}$ ) used for this study should be such that patient can tolerate it well without regurgitation or aspiration and the dilated achalasic esophagus can be filled adequately. It is better to have a fixed volume as a standard protocol. Left posterior oblique films are taken 1, 2 and 5 minutes after barium ingestion (Fig. 2). Usually, three-on-one spot radiographs of $14 \times 14$ or $14 \times 17$ inches size are taken. The distance between the fluoroscope carriage and the patient is kept constant during all three spot films. The 2-minute radiograph is optional, but fluoroscopy is done at 2-minute to see the state of esophageal emptying. If barium completely clears from the esophagus on the 2-minute film, the 5-minute film may be omitted. ${ }^{3}$ One can choose smaller volume of barium suspension especially for pediatric patients. For sequential studies before and after treatment for achalasia, one should consume the same volume of barium as ingested for the baseline examination to have consistent results. ${ }^{3}$

\section{Commonly Encountered Problems During Timed Barium Esophagogram and Measur- es to Overcome These}

In achalasia patients with a massively dilated esophagus, if the barium column cannot be fit on a three-on-one spot film, a two-on-one spot film should be taken. ${ }^{3}$ If the height of the barium column is not fitting length-wise on one film, a spot film is exposed centered over the lower portion of esophagus, followed immediately with another spot film centered over the upper portion of the esophagus. Subsequently, a fixed point is located on each film (usually a vertebral body) that serves as a reference point for both the films. The barium column above or below the reference point is measured on the respective films and added to estimate the height of the entire barium column. In patients with vigorous achalasia, exposing the spot film at a time when the barium column is continuous is difficult due to prominent tertiary esophageal contractions. ${ }^{3}$ In such situation, film should be ex-

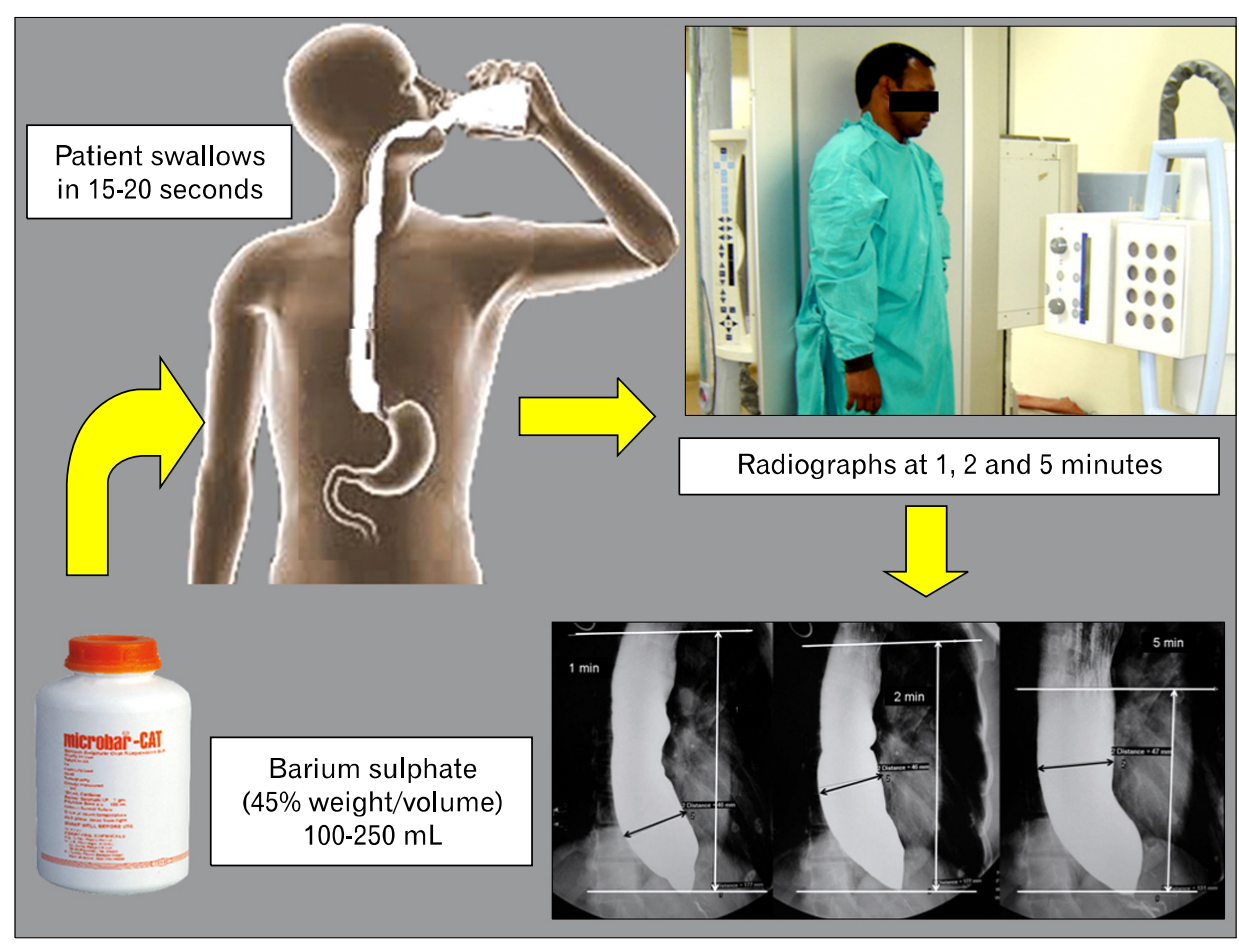

Figure 1. Technique of timed barium esophagogram. Barium sulphate suspension ( $45 \%$ weight/volume) is swallowed by patient in standing position. Three radiographs are taken 1,2 and 5 minute later in left posterior oblique position. 

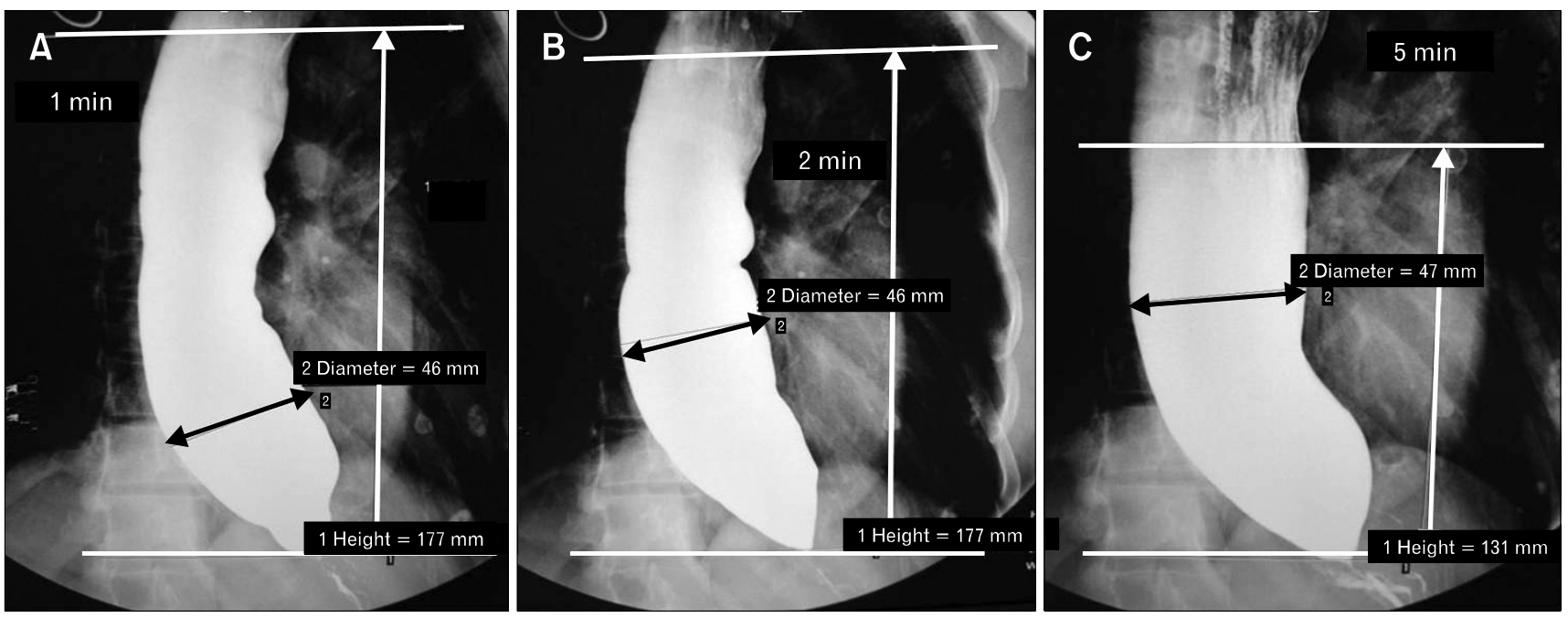

Figure 2. Interpretation of timed barium esophagogram. Radiographs taken 1,2 and 5 minute after ingestion of $150 \mathrm{~mL}$ barium sulphate suspension by an achalasia patient. Two horizontal parallel lines are drawn to measure the height of barium column as shown. Width of barium column is indicated by the transverse black line.
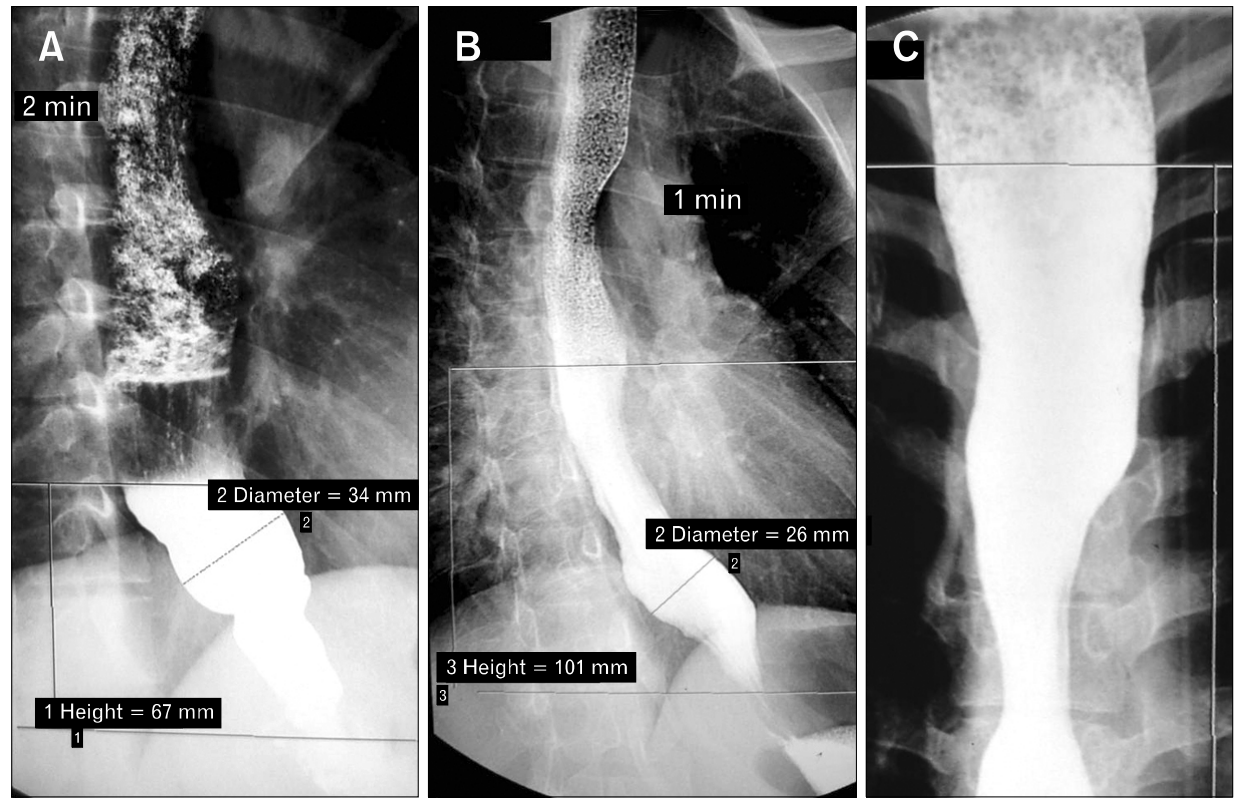

Figure 3. Measurement of height of barium column in presence of bariumfoam interface ( 3 different cases). The superior aspect of the barium column is measured at a point where the margin is reasonably well defined.

posed when the esophagus is relaxed. In patients with achalasia, the esophagus usually contains retained food material and secretions, which may form a barium-foam interface after the patient ingests the barium. In such situation, objectively measuring the height of the barium column may be difficult. When a barium-foam interface forms, one should measure the superior aspect of the barium column at a point where the margin is consistent and reasonably well defined (Fig. 3).

\section{Image Interpretation}

The degree of esophageal emptying can be assessed both qualitatively as well as quantitatively. For quantitative assessment, the height of the barium column is measured from its distinct superior level to the esophago-gastric junction. In patients with achalasia, esophagogastric junction is identified by the classic "bird-beak" appearance. Two horizontal parallel lines, one at 
the lowest and the other at the highest barium level are drawn and the distance between the two is measured (Fig. 2). Diameter of the esophagus can also be measured at the widest part of the barium column perpendicular to the long axis of the esophagus. While many authors have used height and width of barium column in sequential films to assess the emptying, others have used the area of the barium column..$^{3-5,9,10}$ The TBE is a reproducible technique for estimating esophageal empting with almost perfect inter-observer agreement. ${ }^{3}$ Barium completely empties from esophagus in 1 minute in most and in 5 minutes in all healthy individuals. $^{11}$

\section{Utility of Timed Barium Esophagogram in Achalasia and Other Conditions}

In achalasia, esophageal aperistalsis with incomplete lower esophageal sphincter (LES) relaxation leads to stasis in esophagus, thus the barium column persists for longer time as compared to healthy person. As the obstruction persists, the esophagus dilates over time (decompensates). Persistence of barium in esophagus and incomplete or partial emptying over 5 minutes of the study along with other features like 'bird beak' appearance of LES and tertiary contractions in body of esophagus help in diagnosis of achalasia, which is however, confirmed by manometry. The aim of management in these patients is to facilitate the esophageal emptying by early and effective treatment, and prevention of further esophageal dilatation. ${ }^{5}$ Severity and number of achalasia-related symptoms may not correlate with the radiographic findings observed on routine imaging. Hence, for the assessment of severity of achalasia, a physiological assessment of esophageal emptying such as TBE is required. ${ }^{12}$

The improvement in esophageal emptying after successful therapy can be more accurately evaluated by performing repeated TBE during follow-up using same pre-treatment protocol (Fig. 4). The ideal goal of treatment in achalasia is to achieve complete esophageal emptying. Such optimal end point, however, may not be achieved due to aperistaltic dilated esophagus. Also, excessive dilation may lead to higher chance of reflux. Therefore, dilation should be optimized to provide adequate esophageal emptying without abnormal reflux. Most authors have used criteria of ' $50 \%$
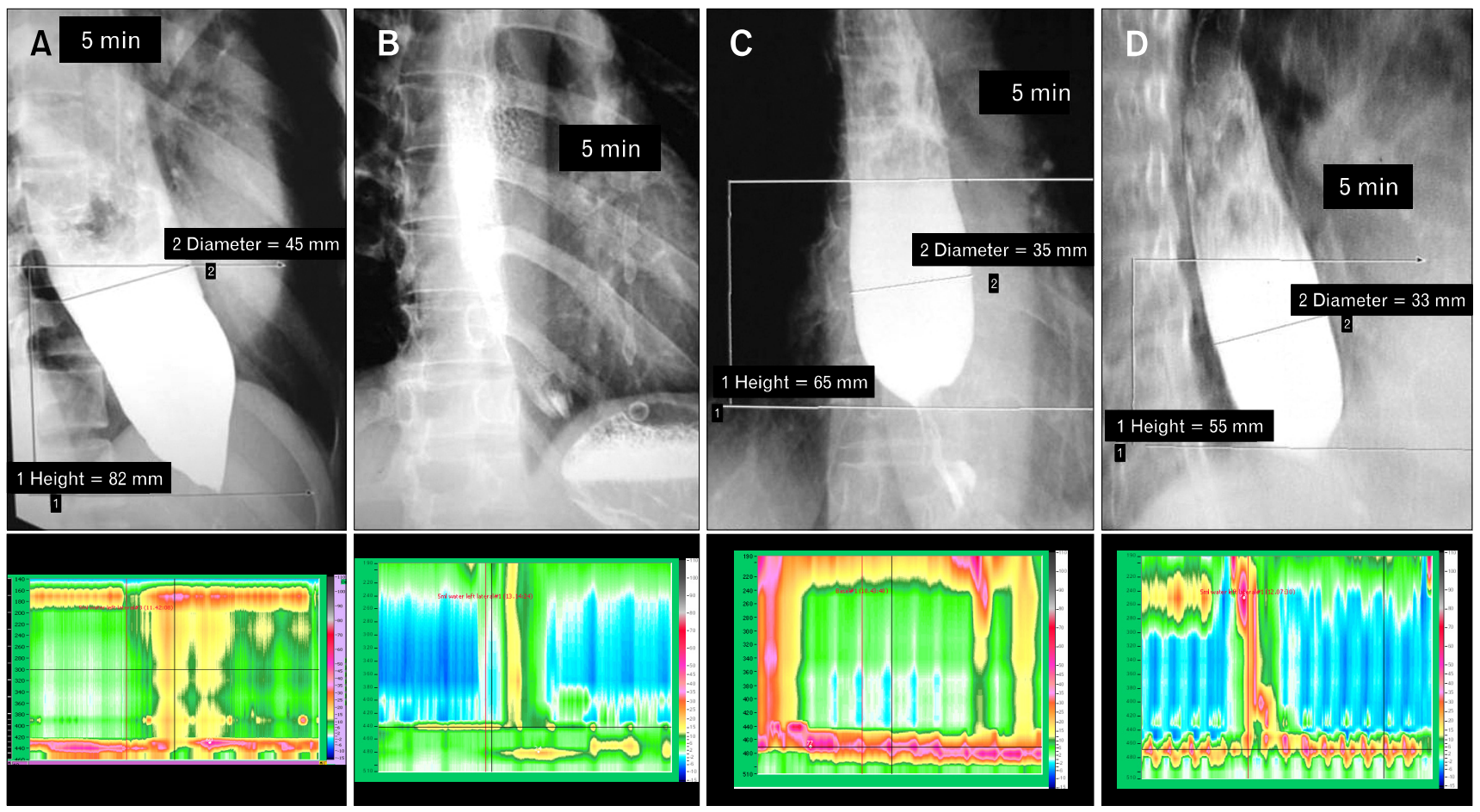

Figure 4. Evaluation of treatment response. Case 1 (A, B): baseline timed barium esophagogram (TBE) shows significant barium retention and high-resolution manometry (HRM) shows high lower esophageal sphincter (LES) pressure. Following pneumatic dilation (PD) patient has complete symptom relief. TBE shows near complete esophageal emptying at 5 minutes and HRM shows relaxation of LES. Case 2 (C, D): baseline and post-dilation images of patient with poor symptomatic improvement following PD. TBE shows only minimal improvement in esophageal emptying at 5 minutes and HRM shows persistent elevated LES pressure suggestive of treatment failure. 
or more reduction in post-treatment height as compared to pre-treatment height on 5-minute film' to define response as per TBE. ${ }^{11}$ Less than $50 \%$ height reduction is taken as failure as per TBE.

Vaezi et $\mathrm{al}^{9,11}$ used TBE for assessment of esophageal emptying following $\mathrm{PD}$ in patients with achalasia. The mean pre-treatment barium height and width were 17.0 and $6.0 \mathrm{~cm}$, respectively. In $72 \%$ of patients, symptoms as well as barium height were improved. Lack of symptomatic improvement was highly associated with poor esophageal emptying $(<50 \%$ improvement in barium height). In this study, in $30 \%$ patients with near complete symptom resolution, esophageal emptying was poor; and most of them had recurrent symptoms within one year. ${ }^{9}$ There may also be mismatch in response as per TBE, manometry and clinical assessment. In patients with significant pre-PD dysphagia, a minor improvement in emptying may be over-interpreted as significant clinical improvement without positive result on $\mathrm{TBE}$; these patients are likely to experience recurrent symptom, which is predicted early by objective assessment on TBE. The patients with good emptying ( $>50 \%$ ) following $\mathrm{PD}$ on TBE are unlikely to report significant and persistent dysphagia. Thus, TBE proved to be a good objective tool for predicting long term response following $\mathrm{PD}$.

Kostic et $\mathrm{al}^{5}$ compared pre- and post-myotomy height and width of barium column in relation to symptom improvement following treatment. Before myotomy, median height of barium column at 1,2 and 5 minutes, were 19,17 and $15 \mathrm{~cm}$, and width were $5.2,4.8$ and $4.5 \mathrm{~cm}$, respectively. Severity of dysphagia before myotomy inversely correlated with change in barium width between 1 to 5-minute radiographs, whereas, regurgitation was related with the height of the barium column. ${ }^{5}$ Successful treatment by myotomy resulted in reduced height and width of the barium column. Post-myotomy height and width of barium column were related to pre-myotomy width; lesser the esophageal dilatation before myotomy, the greater was the improvement in height and width following myotomy. Post-myotomy symptoms correlated poorly with the TBE findings, therefore, poorly assessed success of therapy. Oezcelik et $\mathrm{al}^{10}$ reported that if there was less than $40 \%$ improvement in esophageal clearance following surgical myotomy, moderate to severe dysphagia persisted more often. Rohof et $\mathrm{al}^{13}$ observed that esophageal stasis was a good predictor of treatment failure in long-standing achalasia and proposed use of TBE rather than esophageal manometry to decide on re-treatment. Other studies have also shown that TBE is as good as high resolution manometry to predict response to $\mathrm{PD}$ in patients with achalasia. $^{14,15}$

Baker et $\mathrm{al}^{4}$ used TBE as an initial step in the evaluation of patients with gastroesophageal reflux disease. The rationale behind this was that some patients with symptoms of gastroesophageal reflux disease actually had a misdiagnosed motility disorder. Assessment of motility was performed if TBE showed emptying problem. Patient presenting with liquid dysphagia after anti-reflux surgery also underwent a TBE. Delay in emptying suggested inappropriately tight fundoplication or peristaltic dysfunction.

\section{Conclusion}

Timed barium esophagogram is a simple and objective method for assessing the esophageal emptying. Therefore, TBE should be used in patients with suspected or confirmed achalasia and to follow-up those who have been treated with PD, botulinum toxin injection and Heller myotomy.

\section{References}

1. Levine MS, Rubesin SE. Diseases of the esophagus: diagnosis with esophagography. Radiology 2005;237:414-427.

2. Allen BC, Baker ME, Falk GW. Role of barium esophagography in evaluating dysphagia. Cleve Clin J Med 2009;76:105-111.

3. de Oliveira JM, Birgisson S, Doinoff C, et al. Timed barium swallow: a simple technique for evaluating esophageal emptying in patients with achalasia. AJR Am J Roentgenol 1997;169:473-479.

4. Baker ME, Einstein DM, Herts BR, et al. Gastroesophageal reflux disease: integrating the barium esophagram before and after antireflux surgery. Radiology 2007;243:329-339.

5. Kostic SV, Rice TW, Baker ME, et al. Timed barium esophagogram: a simple physiologic assessment for achalasia. J Thorac Cardiovasc Surg 2000;120:935-943.

6. Chung JJ, Park HJ, Yu JS, et al. A comparison of esophagography and esophageal transit scintigraphy in the evaluation of usefulness of endoscopic pneumatic dilatation in achalasia. Acta Radiol 2008;49: 498-505.

7. Goldenberg SP, Burrell M, Fette GG, Vos C, Traube M. Classic and vigorous achalasia: a comparison of manometric, radiographic, and clinical findings. Gastroenterology 1991;101:743-748.

8. Ghoshal UC, Rangan M, Misra A. Pneumatic dilation for achalasia cardia: reduction in lower esophageal sphincter pressure in assessing response and factors associated with recurrence during long-term follow up. Dig Endosc 2012;24:7-15.

9. Vaezi MF, Baker ME, Achkar E, Richter JE. Timed barium oesophagram: better predictor of long term success after pneumatic dilation in achalasia than symptom assessment. Gut 2002;50:765-770.

10. Oezcelik A, Hagen JA, Halls JM, et al. An improved method of assessing esophageal emptying using the timed barium study following surgical myotomy for achalasia. J Gastrointest Surg 2009;13:14-18. 
11. Vaezi MF, Baker ME, Richter JE. Assessment of esophageal emptying post-pneumatic dilation: use of the timed barium esophagram. Am J Gastroenterol 1999;94:1802-1807.

12. Blam ME, Delfyett W, Levine MS, Metz DC, Katzka DA. Achalasia: a disease of varied and subtle symptoms that do not correlate with radiographic findings. Am J Gastroenterol 2002;97:19161923.

13. Rohof WO, Lei A, Boeckxstaens GE. Esophageal stasis on a timed barium esophagogram predicts recurrent symptoms in patients with long-standing achalasia. Am J Gastroenterol 2013;108:49-55.

14. Gupta M, Ghoshal UC, Verma A, et al. Timed barium esophagogram and high resolution manometry for assessment of response to pneumatic dilation for achalasia cardia: A comparative study [abstract]. J Gasteroenterol Hepatol 2012;27(suppl 5):58.

15. Ghoshal UC, Rangan M. A review of factors predicting outcome of pneumatic dilation in patients with achalasia cardia. J Neurogastroenterol Motil 2011;17:9-13. 